\title{
ON THE METRICAL THEORY OF CONTINUED FRACTIONS
}

\author{
R. NAIR
}

(Communicated by William Adams)

ABstract. Suppose $b_{k}$ denotes either $\phi(k)$ or $\phi\left(p_{k}\right)(k=1,2, \ldots)$ where the polynomial $\phi$ maps $\mathbb{N}$ to $\mathbb{N}$ and $p_{k}$ denotes the $k$ th rational prime. Suppose $\left(c_{k}(x)\right)_{k=1}^{\infty}$ denotes the sequences of partial quotients of the continued function expansion of the real number $x$. Then for certain functions $F: \mathbb{R}_{\geq 0} \rightarrow \mathbb{R}$ we show that

$$
\lim _{N \rightarrow \infty} F^{-1}\left[\frac{F\left(c_{b_{1}}(x)\right)+\cdots+F\left(c_{b_{k}}(x)\right)}{N}\right]=F^{-1}\left[\frac{1}{(\log 2)} \int_{0}^{1} \frac{F\left(c_{1}(x)\right)}{1+x} d x\right]
$$

almost everywhere with respect to Lebesgue measure. This result with $b_{k}=k$ is classical and due to Ryll-Nardzewski.

In this note we consider the continued fraction expansion of a real number $x$ :

$$
x=c_{0}+\frac{1}{c_{1}+\frac{1}{c_{2}}}
$$

Suppose $(X, \beta, \mu, T)$ is a measure-preserving dynamical system. Following [1] we say a strictly increasing sequence of nonnegative integers $\left(b_{n}\right)_{n=1}^{\infty}$ is $L^{p_{-}}$ good universal if for each function $f$ in $L^{p}(X, \beta, \mu)$

$$
\lim _{n \rightarrow \infty} \frac{1}{N} \sum_{n=1}^{N} f\left(T^{b_{n}} x\right)=l(x),
$$

exists $\mu$ almost everywhere.

We prove

Theorem 1. Suppose the function $F: \mathbb{R}_{\geq 0} \rightarrow \mathbb{R}$ is continuous, increasing, and that for some $p \geq 1$ we have

$$
\int_{0}^{1} \frac{\left|F\left(c_{1}(x)\right)\right|^{p}}{x+1} d x<\infty
$$

Received by the editors October 7, 1991 and, in revised form, July 22, 1992. 1991 Mathematics Subject Classification. Primary 11K50; Secondary 28D99. 
For each $n$ in $\mathbb{N}$ and arbitrary nonnegative real numbers $a_{1}, \ldots, a_{n}$ we define

$$
M_{F, n}\left(a_{1}, \ldots, a_{n}\right)=F^{-1}\left[\frac{F\left(a_{1}\right)+\cdots+F\left(a_{n}\right)}{n}\right] .
$$

Assume $\left(b_{n}\right)_{n=1}^{\infty}$ is any strictly increasing sequence of nonnegative integers satisfying the following two conditions:

(i) For each irrational number $\alpha$, if $\langle x\rangle$ denotes the fractional part of $x$, then $\left(\left\langle b_{n} \alpha\right\rangle\right)_{n=1}^{\infty}$ is uniformly distributed modulo one.

Then

(ii) The sequence $\left(b_{n}\right)_{n=1}^{\infty}$ is $L^{p}$-good universal.

$$
\lim _{n \rightarrow \infty} M_{F, n}\left(c_{b_{1}}(x), \ldots, c_{b_{n}}(x)\right)=F^{-1}\left[\frac{1}{\log 2} \int_{0}^{1} \frac{F\left(c_{1}(x)\right)}{1+x} d x\right]
$$

almost everywhere with respect to Lebesgue measure.

The special case $b_{n}=n$ of this result is due to Ryll-Nardzewski [9] who unified earlier work of Khinchine [4]. More general examples of sequences $\left(b_{n}\right)_{n=1}^{\infty}$ satisfying (i) and (ii) include, if $\phi$ denotes an arbitrary nonconstant polynomial mapping $\mathbb{N}$ to itself, $b_{n}=\phi(n)$ and $b_{n}=\phi\left(p_{n}\right)$. Here $p_{n}$ denotes the $n$th rational prime. That both these examples satisfy (i) is well known [11, $8]$. That they satisfy (ii) for any $p>1$ is shown in $[2,6]$.

We mention some applications of Theorem 1 which follow for appropriate choice of $F$.

Corollary 2. If the sequence $\left(b_{n}\right)_{n=1}^{\infty}$ satisfies (i) and (ii) then

$$
\lim _{n \rightarrow \infty} \frac{1}{N} \sum_{k=1}^{N} c_{b_{k}}(x)=\infty
$$

almost everywhere with respect to Lebesgue measure.

Corollary 3. If the sequence $\left(b_{n}\right)_{n=1}^{\infty}$ satisfies (i) and (ii) then

$$
\lim _{N \rightarrow \infty}\left(c_{b_{1}}(x) \cdots c_{b_{N}}(x)\right)^{1 / N}=\prod_{k=1}^{\infty}\left(1+\frac{1}{k^{2}+2 k}\right)^{(\log k) / \log 2}
$$

almost everywhere with respect to Lebesgue measure.

Corollary 4. Suppose the sequence $\left(b_{n}\right)_{n=1}^{\infty}$ satisfies (i) and (ii). Let $P_{N}(x, q)$ $(N=1,2, \ldots)$ denote the number of $c_{b_{1}}(x), \ldots, c_{b_{N}}(x)$ such that if $c_{b_{i}}(x)=q$ then

$$
\lim _{N \rightarrow \infty} \frac{P_{N}(x, q)}{N}=\frac{1}{(\log 2)} \log \frac{(q+1)^{2}}{q(q+2)}
$$

almost everywhere with respect to Lebesgue measure.

We remark that Corollary 2 follows by applying Theorem 1 to $F(x)=x$. Strictly speaking this function does not satisfy the hypothesis of Theorem 1 , however, setting $F(x)=\min (f(x), M)$ and letting $M \rightarrow \infty$ overcomes this difficulty. See the argument leading to (7) for more details. Corollaries 3 and 4 are straightforward.

All these corollaries are known in the case $b_{n}=n$. In this special case Corollaries 2 and 3 are due to Khinchine [4] and Corollary 4 is due to Lévi [5]. 
We say a transformation $T$ of a probability space $(X, \beta, \mu)$ is weakly mixing if for each pair of sets $A$ and $B$ in the sigma-algebra $\beta$ we have

$$
\lim _{N \rightarrow \infty} \frac{1}{N} \sum_{k=1}^{N}\left|\mu\left(A \cap T^{-k} B\right)-\mu(A) \mu(B)\right|=0 .
$$

Let $U$ be the Koopman unitary operator on $L^{2}(X, \beta, \mu)$ defined pointwise by $f(x) \rightarrow f(T x)$. We remind the reader that by approximating arbitrary $L^{2}$ functions $f$ and $g$ by linear combinations of step functions we see that (1) is equivalent to the formally stronger statement

$$
\lim _{N \rightarrow \infty} \frac{1}{N} \sum_{k=1}^{N}\left|\left\langle U^{k} f, g\right\rangle-\langle f, 1\rangle\langle g, 1\rangle\right|=0 .
$$

Here $\langle$,$\rangle denotes the standard inner product on L^{2}(X, \beta, \mu)$.

To prove Theorem 1 we need some lemmas.

Lemma 5. Suppose $\left(f_{n}\right)_{n=1}^{\infty}$ is a bounded nonnegative sequence of real numbers. Then the following two statements are equivalent:

(i) $\sum_{n=1}^{N} f_{n}=o(N)$ and

(ii) $\sum_{n=1}^{N} f_{n}^{2}=o(N)$.

Proof. In [7] following Koopman and von Neumann it is shown that (i) is equivalent to the statement that there exists $M \subseteq \mathbb{N}$ such that

$$
\lim _{N \rightarrow \infty} \frac{\#\{M \cap[1, N]\}}{N}=1 \text { and } \lim _{\substack{n \rightarrow \infty \\ n \in M}} f_{n}=0 .
$$

The equivalence of (i) and (ii) follows immediately from this.

We say a sequence $\left(a_{n}\right)_{n=-\infty}^{\infty}$ is positive definite if for any sequence $\left\{z_{n}\right\}_{n=-\infty}^{\infty}$ having only finitely many nonzero terms we have

$$
\sum_{n, m} a_{n-m} z_{n} \bar{z}_{m} \geq 0
$$

We have the Herglotz theorem [3, p. 38].

Lemma 6. A sequence $\left(a_{n}\right)_{n=-\infty}^{\infty}$ is positive definite if and only if there exists a positive measure $\mu$ on $\mathbb{T}$ such that

$$
a_{n}=\int_{\mathbb{T}} e^{-i n t} d \mu \quad(n \in \mathbb{Z})
$$

(i.e., the nth Fourier coefficient of $\mu$ denoted by $\hat{\mu}(n))$.

We now have Wiener's criteria [3, p. 42] for the continuity of a measure.

Lemma 7. A measure $\mu$ on $\mathbb{T}=\mathbb{R} / 2 \pi \mathbb{Z}$ has no atoms if and only if

$$
\lim _{n \rightarrow \infty} \frac{1}{2 n+1} \sum_{-N}^{N}|\hat{\mu}(n)|^{2}=0 .
$$

Lemma 8. Suppose that the measure-preserving dynamical system $(X, \beta, \mu, T)$ is weakly mixing and that the strictly increasing sequence of nonnegative integers 
$\left(\dot{b}_{n}\right)_{n=1}^{\infty}$ satisfies conditions (i) and (ii). Then if $f \in L^{p}(X, \beta, \mu)$ with $p>1$ we have $l(x)=\int_{X} f d \mu \quad \mu$ almost everywhere.

Proof. We begin by proving the statement in norm, that is,

$$
\lim _{N \rightarrow \infty}\left\|\frac{1}{N} \sum_{k=1}^{N} f\left(T^{b_{k}} x\right)-\int_{X} f d \mu\right\|=0,
$$

where $\|f\|$ denotes the $L^{2}(X, \beta, \mu)$ norm of the function $f$. By arguing with $f(x)-\int_{X} f d \mu$ instead of $f(x)$ if necessary, we can assume without loss of generality that $\int_{X} f d \mu=0$. Now let $U$ denote the Koopman linear isometry on $L^{2}(X, \beta, \mu)$. As is immediately verified, letting $U^{n}$ denote $\left(U^{*}\right)^{|n|}$ if $n<0$, the sequence $\left(\left\langle U^{n} f, f\right\rangle\right)_{n=-\infty}^{\infty}$ is positive definite; hence, there is a measure $\omega_{f}$ on $\mathbb{T}$, dependent on $f$ such that

$$
\left\langle U^{n} f, f\right\rangle=\int_{\mathbb{T}} e^{-i n t} d \omega_{f}(t) \quad(n \in \mathbb{Z}) .
$$

Some basic properties of $U$ can be found in [10, p. 25]. This means that

$$
\begin{aligned}
\left\|\frac{1}{N+1} \sum_{k=0}^{N} f\left(T^{b_{k}} x\right)\right\|^{2} & =\frac{1}{(N+1)^{2}} \sum_{0 \leq k_{1}, k_{2} \leq N}\left\langle U^{b_{k_{2}}-b_{k_{1}}} f, f\right\rangle \\
& =\frac{1}{(N+1)^{2}} \sum_{0 \leq k_{1}, k_{2} \leq N} \int_{0}^{1} e^{2 \pi i\left(b_{k_{1}}-b_{k_{2}}\right) \theta} d \omega_{f}(\theta) \\
& =\int_{0}^{1}\left|\frac{1}{N+1} \sum_{k=0}^{N} e^{2 \pi i b_{k} \theta}\right|^{2} d \omega_{f}(\theta) .
\end{aligned}
$$

By Lemma 7 the measure $\omega_{f}$ is nonatomic if we can show that

$$
\lim _{N \rightarrow \infty} \frac{1}{N} \sum_{k=1}^{N}\left|\hat{\omega}_{f}(n)\right|^{2}=0 .
$$

Because $\left|\hat{\omega}_{f}(n)\right| \leq\left\|\omega_{f}\right\|$ for each $n \in \mathbb{Z}$, by Lemma 5 , it is sufficient to show

$$
\lim _{N \rightarrow \infty} \frac{1}{N} \sum_{k=1}^{N}\left|\hat{\omega}_{f}(n)\right|=0 .
$$

Now note that the weak-mixing of $T$ in the form (2) and the fact that $\int_{X} f d \mu=$ 0 give

$$
\lim _{N \rightarrow \infty} \frac{1}{N} \sum_{k=1}^{N}\left|\hat{\omega}_{f}(n)\right|=\lim _{N \rightarrow \infty} \frac{1}{N} \sum_{k=1}^{N}\left|\left\langle U^{k} f, f\right\rangle\right|=0 .
$$

This means $\omega_{f}$ is nonatomic. It now follows that (3) holds using (4) and (i), that is, the fact that $\left(\left\langle b_{k} \theta\right\rangle\right)_{k=1}^{\infty}$ is uniformly distributed modulo one for each irrational $\theta$.

To prove the almost everywhere convergence we argue as follows. Let $\left(N_{t}\right)_{t=1}^{\infty}$ denote a strictly increasing sequence of integers such that

$$
\left\|\frac{1}{N_{t}} \sum_{k=1}^{N_{t}} f\left(T^{b_{k}} x\right)-\int_{X} f d \mu\right\|<\frac{1}{t} .
$$


Thus

$$
\sum_{t=1}^{\infty}\left\|\frac{1}{N_{t}} \sum_{k=1}^{N_{t}} f\left(T^{b_{k}} x\right)-\int_{X} f d \mu\right\|^{2}<\infty .
$$

Hence rearranging using Fatou's lemma we have

$$
\int_{X}\left(\sum_{t=1}^{\infty} \| \frac{1}{N} \sum_{k=1}^{N_{t}} f\left(T^{b_{k}} x\right)-\left.\int_{X} f d \mu\right|^{2}\right) d \mu<\infty .
$$

Thus using the fact that if $\sum a_{n}<\infty$ with $a_{n} \geq 0$ theri $a_{n}=o(1)$, we have

$$
\lim _{t \rightarrow \infty} \frac{1}{N_{t}} \sum_{k=1}^{N_{t}} f\left(T^{b_{k}} x\right)=\int_{X} f d \mu \text { a.e. }
$$

By (ii), that is, the $L^{p}$-good universality property, the limit exists and so it must be $\int_{X} f d \mu$ as required by (5).

We now complete the proof of Theorem 1. Let $T$ denote the map of $(0,1)$ defined by $T x=\langle 1 / x\rangle$. We note that the partial quotients $\left(c_{k}(x)\right)_{k=1}^{\infty}$ of $x$ satisfy $c_{k}(x)=c_{1}\left(T^{k-1} x\right)$. Gauss observed that the map $T$ preserves the measure

$$
\mu(A)=\frac{1}{\log 2} \int_{A} \frac{d x}{1+x} .
$$

This measure is equivalent to Lebesgue measure on $[0,1]$ (in the sense that it has the same null sets). In addition the transformation $T$ is weak mixing on $[0,1]$ with respect to the measure $\mu$ in (6). If $\int_{0}^{1} F\left(c_{1}(x)\right) d x /(1+x)$ is finite then Theorem 1 follows immediately from Lemma 8. If $\int_{0}^{1} F\left(c_{1}(x)\right) d x /(1+x)$ is not finite, we argue as follows. Let

$$
f_{M}(x)= \begin{cases}F\left(c_{1}(x)\right) & \text { if }\left|F\left(c_{1}(x)\right)\right| \leq M \\ M & \text { if }\left|F\left(c_{1}(x)\right)\right|>M\end{cases}
$$

This means that for each $M \geq|F(1)|$ and for almost all $x$

$$
\begin{aligned}
\lim _{N \rightarrow \infty} \inf \frac{1}{N} \sum_{k=1}^{N} F\left(c_{b_{k}}(x)\right) & \geq \liminf _{N \rightarrow \infty} \frac{1}{N} \sum_{k=1}^{N} f_{M}\left(T^{b_{k}-1} x\right) \\
& =\frac{1}{(\log 2)} \int_{0}^{1} \frac{f_{M}(x)}{1+x} d x .
\end{aligned}
$$

Now letting $M$ tend to infinity completes the proof.

\section{REFERENCES}

1. A. Bellow and V. Losert, The weighted pointwise ergodic theorem and the individual ergodic theorem along subsequences, Trans. Amer. Math. Soc. 288 (1985), 307-345.

2. G. A. Edgar and L. Sucheston (eds.), Almost everywhere convergence, Academic Press, New York, 1989, pp. 145-151.

3. Y. Katznelson, An introduction to harmonic analysis, Dover, New York, 1976.

4. A. Khinchine, Metrische Kettenbruch probleme, Compositio Math. 1 (1935), 359-382. 
5. P. Lévi, Sur le dévelopment en fraction continue d'un nombre choise au hasard, Compositio Math. 3 (1936), 286-303.

6. R. Nair, On polynomials in primes and J. Bourgain's circle method approach to ergodic theorems. II, Studia Math. (to appear).

7. K. Peterson, Ergodic theory, Graduate Stud. in Adv. Math., vol. 2, Cambridge Univ. Press, London and New York, 1981.

8. G. Rhin, Répartition modulo 1 de $f\left(p_{n}\right)$ quand $f$ est une series entiere, Lecture Notes in Math., vol. 475, Springer-Verlag, New York, 1975, pp. 176-244.

9. C. Ryll-Nardzewski, On the ergodic theorems. II: Ergodic theory of continued fractions, Studia Math. 12 (1951), 74-79.

10. P. Walters, An introduction to ergodic theory, Graduate Texts in Math., vol. 79, SpringerVerlag, New York, 1982.

11. H. Weyl, Über die Gleichvertilung von Zahlen mod. Eins, Math. Ann. 77 (1916), 313-352.

Department of Pure Mathematics, University of Liverpool, P.O. Box 147, Liverpool L69 3BX, UNITED KINGDOM 